\title{
Vortices Solutions in Chern-Simons-Maxwell-Higgs System
}

J. M. Hoff da Silva*

Instituto de Física Teórica (IFT-UNESP), Brazil

E-mail: hoffeift.unesp.br

M. C. B. Abdalla Ribeiro

Instituto de Física Teórica (IFT-UNESP), Brazil

E-mail: mabdalla@ift.unesp.br

\section{E. X. Guimarães}

Universidade de Brasilia (UnB), Brazil

E-mail: marg@unb.br

In this work we analyze some characteristics of Bogomol'nyi's equations for abelian gauge theories with Chern-Simons-Maxwell-Higgs terms. We don't obtain the complete solution for the model, but we analyze the equations in a kind of weak field approximation instead of using a computational analysis. Our main idea is to get some intuition on the subject. Working with the equations in such approach we reproduce the accurate behavior next to the origin.

Fifth International Conference on Mathematical Methods in Physics - IC2006

April 24-28 2006

Centro Brasileiro de Pesquisas Fisicas, Rio de Janeiro, Brazil

${ }^{*}$ Speaker. 


\section{Introduction}

Homotopy groups play a very important role when applied in topological systems. They lead us to a kind of solution - the so-called topological defect of the theory. In the case of abelian gauge systems with Chern-Simons-Maxwell-Higgs terms the nontrivial homotopy group is the fundamental one. Vortices solutions are related with gauge theory by a Higgs field, or to be more precise by a spontaneous symmetry breaking (SSB) mechanism. Besides, when the lagrangian shows SSB and the ground state of a scalar field (vacuum value) is different of zero and the fundamental group is nontrivial we have vortices solutions. This is the case in question. In such abelian system we obtain self-dual vortices equations. For this purpose we first introduce, in the second section, the principal ideas of the model. In the third section we find the self-dual equations and then solve the system in an weak field approximation for the regime $r \rightarrow 0$.

\section{The Model}

Following the work of Choonkyu Lee et al [1], we start with a lagrangian density defined in a $(2+1)$ dimensional flat spacetime given by

$$
\begin{aligned}
L & =-\frac{1}{4} F_{\mu v} F^{\mu v}+\frac{1}{4} \mu \varepsilon^{\mu v \rho} F_{\mu v} A_{\rho} \\
& +\left|D_{\mu} \phi\right|^{2}+\frac{1}{2}\left(\partial_{\mu} N\right)^{2}-V(\phi, N),
\end{aligned}
$$

where $D_{\mu}=\partial_{\mu}-i e A_{\mu}, \phi$ is a scalar complex field and $N$ a neutral scalar field. Clearly, the system is a $U(1)$ gauge theory. The potential of model is

$$
V(\phi, N)=\frac{1}{2}\left(e|\phi|^{2}+\mu N-e a^{2}\right)^{2}+e^{2} N^{2}|\phi|^{2} .
$$

When the symmetry is broken there appears two degenerate states in the energy ground state. However, the most important case, in the sense of topological solutions, shows up when

$$
|\phi|_{\min }^{2}=a^{2}-N(1+\mu / e)
$$
is 1

In the ground state, the system is not invariant by $U(1)$ action, and the first homotopy group

$$
\pi_{1}(U(1) / I)=Z
$$

If we look for self-dual equations first we need to write down the energy functional related to the lagrangian given by eq. (2.1), so

$$
\varepsilon=\int d^{2} x\left(\frac{1}{2} F_{i 0}^{2}+\frac{1}{2} F_{12}^{2}+\left|D_{0} \phi\right|^{2}+|\mathbf{D} \phi|^{2}+\frac{1}{2}\left(\partial_{0} N\right)^{2}+\frac{1}{2}\left(\partial_{i} N\right)^{2}+V\right) .
$$

Here the first and the second terms are the electric and magnetic field contributions, respectively, and the intermediary terms are the Higgs scalar field contribution besides, of course, the neutral field and the potential ${ }^{2}$. The next section is dedicated to obtain the self-dual equations.

\footnotetext{
${ }^{1}$ The complete proof is in the appendix.

${ }^{2}$ Note that there's no contribution from Chern-Simons term. In fact, this term has no dynamics and do not depend on the metric. The energy-momentum tensor of $\frac{1}{4} \mu \varepsilon^{\mu v \rho} F_{\mu v} A_{\rho}$ is zero.
} 


\section{Bogomol'nyi's Equations}

Is well known that this kind of system [6] have the minimum of energy proportional to the magnetic flux ( $\Theta$ in our notation). So, working out equation (2.5) one finds

$$
\begin{aligned}
\varepsilon & =e a^{2}|\Theta|+\int d^{2} x\left(\frac{1}{2} F_{i 0}^{2}+\frac{1}{2} F_{12}^{2}\right. \\
& +\left|D_{0} \phi\right|^{2}+\left|\left(D_{1}+i D_{2}\right) \phi\right|^{2} \\
& \pm e F_{12}|\phi|^{2}+\frac{1}{2}\left(\partial_{0} N\right)^{2}+\frac{1}{2}\left(\partial_{i} N\right)^{2} \\
& +\frac{1}{2}\left(e|\phi|^{2}+\mu N-e a^{2}\right)^{2} \\
& \left.+e^{2} N^{2}|\phi|^{2} \mp e a^{2} F_{12}\right) .
\end{aligned}
$$

Where we made use of a "key equation"

$$
|\mathbf{D} \phi|^{2}=\left|\left(D_{1} \pm i D_{2}\right) \phi\right|^{2} \pm e B|\phi|^{2} \pm \varepsilon^{i j} \partial_{i} J_{j},
$$

without considering the superficial term and writing down explicitly the potential. The Gauss law is given by

$$
-\partial_{i} F_{0 i}+\mu F_{12}-e J_{0}=0
$$

with

$$
J_{0}=-i\left[\phi^{*}\left(D_{0} \phi\right)-\left(D_{0} \phi\right)^{*} \phi\right] .
$$

We want to write the equation (3.1) like a sum of squares. To do it we first note that

$$
\begin{gathered}
\frac{1}{2}\left(F_{i 0} \pm \partial_{i} N\right)^{2}=\frac{1}{2} F_{i 0}^{2} \pm F_{i 0} \partial_{i}+N \frac{1}{2}\left(\partial_{i} N\right)^{2}, \\
\frac{1}{2}\left[F_{12} \pm\left(e|\phi|^{2} \mu N-e a^{2}\right)\right]^{2}=\frac{1}{2} F_{12}^{2} \pm F_{12}\left(e|\phi|^{2} \mu N-e a^{2}\right) \\
\times \frac{1}{2}\left(e|\phi|^{2}+\mu N-e a^{2}\right)^{2}
\end{gathered}
$$

and

$$
\left|D_{0} \phi \mp i e \phi N\right|^{2}=\left|D_{0} \phi\right|^{2}+e^{2} N^{2}|\phi|^{2} \mp e N J_{0} .
$$

With this equations and taking into account the constraint imposed by the Gauss law we have

$$
\begin{aligned}
\varepsilon & =e a^{2}|\Theta|+\int d^{2} x\left(\frac{1}{2}\left(F_{i 0} \pm \partial_{i} N\right)^{2}\right. \\
& +\frac{1}{2}\left[F_{12} \pm\left(e^{2}|\phi|^{2}+\mu N-e a^{2}\right)\right]^{2} \\
& \left.+\left|D_{0} \phi \mp i e \phi N\right|^{2}+\left|\left(D_{1} \pm i D_{2}\right) \phi\right|^{2}+\frac{1}{2}\left(\partial_{0} N\right)^{2}\right) .
\end{aligned}
$$


Now we are able to write the self-dual equations. To this purpose let's first set $\partial_{0} N=0$ (static configuration). We have the minimum energy for the system $\left(\varepsilon=e a^{2}|\Theta|\right)$ when

$$
\begin{gathered}
F_{i 0} \pm \partial_{i} N=0 \\
F_{12} \pm\left(e^{2}|\phi|^{2}+\mu N-e a^{2}\right)=0 \\
D_{0} \phi \mp i e \phi N=0
\end{gathered}
$$

and

$$
\left(D_{1} \pm i D_{2}\right) \phi=0 \text {. }
$$

As we are interested in the static configurations of the fields, we can to reduce the above equations to

$$
\begin{gathered}
e A_{i} \mp \varepsilon_{i j} \partial_{j} \ln |\phi|=0, \\
-\nabla^{2} N+\left(\mu^{2}+2 e^{2}|\phi|^{2}\right) N-e \mu\left(a^{2}-|\phi|^{2}\right)=0
\end{gathered}
$$

and

$$
-\nabla^{2} \ln |\phi|^{2}+2 e\left(e|\phi|^{2}+\mu N-e a^{2}\right)=0 .
$$

At this point we can search for solutions. We shall work in a special configuration of fields called rotationally symmetric, given by the following ansatz

$$
\phi=g(r) \exp (\operatorname{in} \theta)
$$

and

$$
e A_{i}= \pm \frac{\varepsilon_{i j} \hat{r}_{j}}{r}\left(\frac{g^{\prime}}{g}-n\right)
$$

where $n$ is a nonnegative integer and prime means $d g / d r$. Our expectation is that the fields are well defined when $r \rightarrow 0$ and, when $r \rightarrow \infty$ we expect $g \rightarrow a$ and $N \rightarrow 0$, since this boundary conditions gives rise to finite energy solutions.

To solve completely the equations (3.14) and (3.15), together with the ansatz we would need numerical analysis. However, as it was mentioned before we shall use a very convenient approximation. First, let's define $g(r)=\exp (y(r))$ and then pick up only the first (linear) term in the equation (3.15) and after chose, without lost of essential generality, the vacuum field value equal to one. Our approximation consists into analyze the system in the limit of weak fields. In practical terms it means to considerer only linear terms in the expansion of exp $y(r)$ and $N$. So, gathering all these modifications and considerations in the self-dual equations we have

$$
\nabla^{2} N-\left(2 e^{2}+\mu^{2}\right) N-2 \mu e y=0
$$

and

$$
\nabla^{2} y-2 e^{2} y-\mu e N=0
$$


A bit of simple algebra led to the following result

$$
y^{(i v)}+\frac{2}{r} y^{\prime \prime \prime}-\left(\frac{1}{r^{2}}+K_{1}\right) y^{\prime \prime}+\frac{1}{r}\left(\frac{1}{r^{2}}-K_{1}\right) y^{\prime}+K_{2} y=0,
$$

where $K_{1} \equiv 4 e^{2}+\mu^{2}$ and $K_{2} \equiv 4 e^{4}$ are constants. Now the equation (3.20) can be integrated out without computational analysis. The solution is a complicated combination of first and second kind modified Bessel equations given by

$$
y(r)=C_{1} Y_{0}(x(r))+C_{2} J_{0}\left(x^{\prime}(r)\right)+C_{3} Y_{0}\left(x^{\prime}(r)\right)+C_{4} J_{0}(x(r)),
$$

where $J(0)$ and $Y(0)$ are the Bessel's functions (of zero index) of first and secund kind, respectively and

$$
\begin{gathered}
x(r)=\frac{r}{2} \sqrt{-2 K_{1}-2 \sqrt{-4 K_{2}+K_{1}^{2}}} \\
x^{\prime}(r)=\frac{r}{2} \sqrt{-2 K_{1}+2 \sqrt{-4 K_{2}+K_{1}^{2}}} .
\end{gathered}
$$

Let's see now what it happens when $r \rightarrow 0$. In this range the asymptotic behavior of Bessel's functions is

$$
J_{0}(x) \sim 1
$$

and

So, our solution becomes

$$
Y_{0}(x) \sim \frac{2}{\pi} \ln (x)
$$

$$
\begin{aligned}
y & =C_{1} \ln \left[\frac{r}{2} \sqrt{-2 K_{1}-2 \sqrt{-4 K_{2}+K_{1}^{2}}}\right]+C_{3} \ln \left[\frac{r}{2} \sqrt{-2 K_{1}+2 \sqrt{-4 K_{2}+K_{1}^{2}}}\right] \\
& +C_{2}+C_{4}
\end{aligned}
$$

or in terms of scalar field

$$
\begin{aligned}
g & =\left(\frac{r}{2} \sqrt{-2 K_{1}-2 \sqrt{-4 K_{2}+K_{1}^{2}}}\right)^{C_{1}} \\
& \times\left(\frac{r}{2} \sqrt{-2 K_{1} 2 \sqrt{-4 K_{2}+K_{1}^{2}}}\right)^{C_{3}} \\
& \times \exp \left(C_{2}+C_{4}\right) .
\end{aligned}
$$

Adjusting the integration constants $\left(C_{1}=C_{3}=2\right)$ we have a interesting situation

$$
g(r)=4 e^{4} \exp \left(C_{2}+C_{4}\right) r^{4} .
$$

So, the scalar field increases with $r^{4}$ showing up a good behavior (comparing figures 1 and 2). Unfortunately when $r \rightarrow \infty$ the field $g$ goes to zero with a factor $1 / \sqrt{r}$ and it does not saturate to the vacuum value. Then, our approximation fails in this sector. 


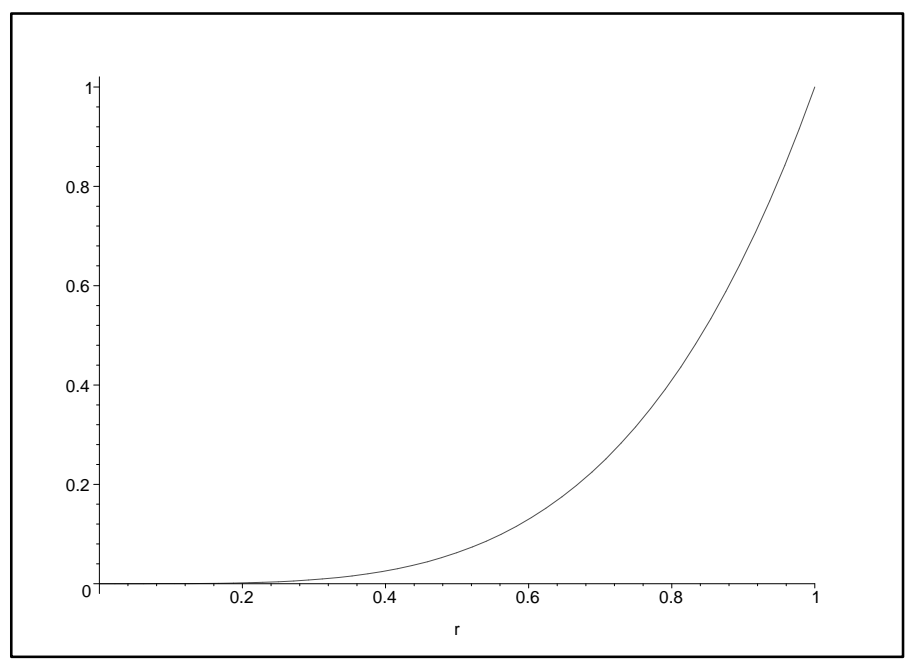

Figure 1: Grafic of $g(r)$ in the range $r \rightarrow 0$, showing up a desirable behavior.

\section{Final Remarks}

The model we considered is the simplest case with Chern-Simons and Maxwell terms together in the lagrangian. A little more complicated model can be find in [1]. The main difference is the presence of another scalar field. However the system worked here gives a good example of planar vortices in abelian gauge theories. Our method of solving the Bogomol'nyi's equations is, again, based upon on a trick to linearize fields equations in an weak field approximation and, then, adjust the integration constants to obtain physical solutions.

The calculation uses a special kind of configuration called rotationally symmetric. In this case we obtain a solution given by second kind modified Bessel's equations. In fact, it's a direct consequence of cylindrical symmetry of the system.

To conclude, we call the attention for the fact of that this trick reproduce all solution for a little bit more simple model with a Chern-Simons-Higgs lagrangian given by

$$
L=\frac{\mu}{4} \varepsilon^{\mu v \rho} A_{\mu} F_{v \rho}+\left|D_{\mu} \phi\right|^{2}-V(|\phi|) .
$$

The energy functional is

$$
\varepsilon=\int d^{2} x\left[\left|\left(D_{1} \pm i D_{2}\right) \phi\right|^{2} \mp e B|\phi|^{2}+\left|D_{0} \phi\right|^{2}+V(|\phi|)\right],
$$

without the surface term. Proceeding from similar way to the previous case we have

$$
\begin{aligned}
\varepsilon & =a^{2}|\Theta|+\int d^{2} x\left(\left|D_{0} \phi\right|^{2}\right. \\
& \pm\left.\frac{i e}{\mu}\left(|\phi|^{2}-a^{2}\right) \phi\right|^{2}+\left|\left(D_{1} \pm i D_{2}\right) \phi\right|^{2} \\
& \left.+V(|\phi|)-\frac{e^{2}}{\mu^{2}}|\phi|^{2}\left(|\phi|^{2}-a^{2}\right)^{2}\right),
\end{aligned}
$$

thus the Bogomol'nyi's equations are

$$
\left(D_{1} \pm i D_{2}\right) \phi=0
$$




$$
D_{0} \phi=\mp \frac{i e}{\mu}\left(|\phi|^{2}-a^{2}\right) \phi
$$

and

$$
V(|\phi|)=\frac{e^{2}}{\mu^{2}}|\phi|^{2}\left(|\phi|^{2}-a^{2}\right)^{2} .
$$

Working once again with rotationally symmetric field configuration and weak approximation the scalar field assumes the form

$$
\begin{aligned}
g & =\exp \left(\left[\ln \left(\frac{r}{2}\right)+\alpha\right] I_{0}(r)+\frac{r^{2}}{2^{2}}\right. \\
& \left.+\frac{r^{4}}{2^{2} \cdot 4^{2}}\left(1+\frac{1}{2}\right) \frac{r^{6}}{2^{2} \cdot 4^{2} \cdot 6^{2}}\left(1+\frac{1}{2}+\frac{1}{3}\right)+\cdots\right)
\end{aligned}
$$

where $\alpha$ is an arbitrary constant and $I_{0}(r)$ is given by

$$
I_{0}(r)=1+\frac{r^{2}}{2^{2}}+\frac{r^{4}}{2^{2} \cdot 4^{2}}+\frac{r^{6}}{2^{2} \cdot 4^{2} \cdot 6^{2}}+\cdots
$$

The behavior of scalar field can be better visualized in the figure 2 . Note the topological stability when $r \rightarrow \infty$ showing a good behavior.

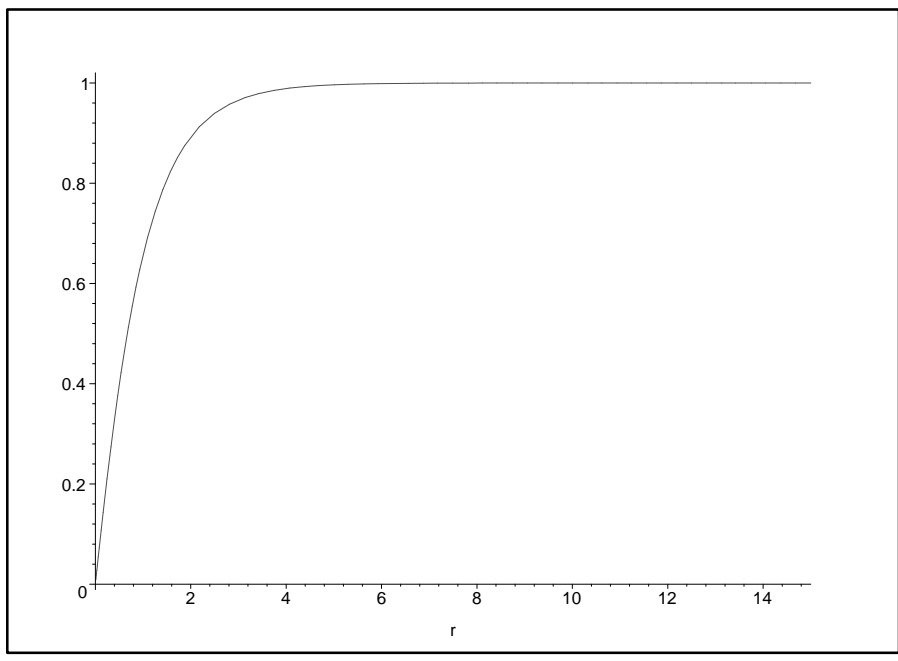

Figure 2: Grafic of $g(r)$ versus $r$. Note the topological stability.

\section{Appendix}

Here we will give a proof of the isomorphism between the circle $S^{1}$ and the additive group of integers $Z\left(\pi_{1}\left(S^{1}\right)=Z\right)$ [8].

Proof.: To each classroom of homotopy $\alpha=[a]$ of closed paths in $S^{1}$ we will associate the entire $n(a)$. The degree $n(a)$ depends only on the classroom but $\alpha$ not of the closed way $a$ that we choose to represent it. Thus we can speak in the degree $n(\alpha)$ of classroom $\alpha$ and get an application $n: \pi_{1}\left(S^{1}\right) \rightarrow Z$. Since that $n$ is a homomorphism bijective we have an isomorphism of $\pi_{1}\left(S^{1}\right)$ on $Z$. 
M.C.B.A. and M.E.X.G. acknowledge CNPq for partial support and J.M.H.S. would like to acknowledge FAPESP for financial support.

\section{References}

[1] Choonkyu Lee, Kimyeong Lee and Hyunsoo Min, Self-dual Maxwell Chern-Simons solitons, Phys. Lett. B 252, 79 (1990);

[2] R. Jackiw and E. Weinberg, Self-Dual Chern Simons Vortices, Phys. Rev. Lett. 64, 2234 (1990);

[3] R. Jackiw, K. Lee and E. Weinberg, Self-dual Chern-Simons solitons, Phys. Rev. D 42, 3466 (1990);

[4] J. Hong, Y. Kim and P. Y. Pac, Multivortex solutions of the Abelian Chern-Simons-Higgs theory, Phys. Rev. Lett. 64, 2230 (1990);

[5] Kimyeong Lee, Relativistic nonabelian self-dual Chern-Simons systems, Phys. Lett. B 255, 381 (1991);

[6] N. K. Nielsen and P. Olesen, Vortex-line models for dual strings, Nuclear Physics B61 45 (1973);

[7] E. B. Bogomol'nyi, The stability of classical solutions, Sov. J. Nucl. Phys. 24, 449 (1976);

[8] E. L. Lima, Grupo fundamental e espaços de recobrimento, IMPA (1998). 